\title{
Confining Potential and Mass of Elementary Particles
}

\author{
Lev I. Buravov \\ Institute of Problems of Chemical Physics RAS, Chernogolovka, Russia \\ Email: buravov@icp.ac.ru
}

Received 14 July 2015; accepted 18 January 2016; published 22 January 2016

Copyright (C) 2016 by author and Scientific Research Publishing Inc.

This work is licensed under the Creative Commons Attribution International License (CC BY).

http://creativecommons.org/licenses/by/4.0/

(c) (i) Open Access

\begin{abstract}
In this paper we consider a model in which the masses of elementary particles are formed and stabilized thanks to confining potential, which is caused by recoil momentum at emission of specific virtual bosons by particle itself. The calculation of this confining potential $\Phi(R)$ is carried out. It is shown that $\Phi(R)$ may be in the form const $R^{3}$ or const $R^{2}$ depending on continuous or discrete nature of the spectrum of emitted bosons.
\end{abstract}

\section{Keywords}

Confining Potential, Origin of Mass of Particle, Stabilizing of Particle Mass, Virtual Bosons, Spherical Bosonic Wave, Origin of Neutrino Masses, Neutrino Mass Formula, Calculation of Neutrino Masses

\section{Introduction}

In the article the model of formation of mass of elementary particles is offered as a result of emitting of the special virtual bosons as spherical waves (conditionally we will name them as the bosons of Higgs). It is supposed here that confining potential which is necessary for stabilizing of particle mass appears because of effect of impulse recoil, especially for electron, muon, pion, kaon and neutrino.

In due time Poincare, proceeding from common sense, entered supposition about a presence in the structure of electron of some elastic elements due to which the charge of electron holds out in a small volume. This model was later used by many authors. We will consider it more detailed, following [1] [2].

In accordance with [1] [2] virtual rest energy of electron $E$ consists of two parts: surface energy of elastic shell $W_{s}=\sigma 4 \pi R^{2}$ and electrostatic energy of the charged surface $W_{e}=e^{2} / 2 R$, where $\sigma$ is the coefficient of surface tension of the shell, $R$ is its radius, $e^{2}=q^{2} / 4 \pi \varepsilon_{0}, \varepsilon_{0}$ is dielectric constant of vacuum, $q$ is an electron charge (in units of SI). Virtual rest energy of such system is equal to: 


$$
E=\sigma 4 \pi R^{2}+e^{2} / 2 R
$$

The radius of electron, corresponding to a minimum of energy (1) of the system, is determined from equation $\partial E / \partial R=0$ and is equal to:

$$
R_{e}=0.5\left(e^{2} / 2 \pi \sigma\right)^{1 / 3}
$$

From Equations (1) and (2) the mass of electron is

$$
m_{e}=3(\pi \sigma / 4)^{1 / 3} e^{4 / 3} / c^{2}
$$

It is possible also to write down that $m_{e}=12 \sigma \pi R_{e}^{2} / c^{2}=f R_{e}^{2}$, where a $\sigma$ value was determined in [3] with using the value of neutral pion mass $m_{0}=134.963 \mathrm{MeV} / \mathrm{c}^{2}$ :

$$
\sigma=4 \times 3^{-7} \pi^{-3} \hbar^{-2} c^{4} m_{0}^{3}=5.967 \times 10^{14} \mathrm{~J} / \mathrm{m}^{2}
$$

and coefficient $f=12 \sigma \pi / \mathrm{c}^{2} \cong 1.404 \times 10^{25} \mathrm{MeV} / \mathrm{cm}^{2}$.

In Equation (1) the value $W_{s}=\sigma 4 \pi R^{2}$ obviously fulfills the role of confining potential due to which the mass of electron is stabilized.

Preliminary we will represent information about the calculation of the masses for several elementary particles and then pass to more detailed consideration of confining potential.

In [3] the calculation of the ratio for the masses of the particles $e, \mu, \pi^{0}, \pi^{ \pm}, K^{0}, K^{ \pm}$was made on the basis of starting model assumption that the stopped muon, pion and kaon can be represented as spherical resonators for quanta of virtual neutrinos excited into an elastic lepton shell with surface energy $W_{s}=\sigma 4 \pi R^{2}$, where $R$ is a radius of elastic shell, $\sigma$ is the coefficient of surface tension, the same that is in Equations (1)-(4). In [3] it was shown that virtual rest energy of these particles can be written down in general case in some more complex forms, than those for an electron:

$$
E=\sigma 4 \pi R^{2}+1.5 N \hbar \pi c /(R-\rho)+e^{2} / 2 \rho
$$

where $\rho$ is a radius of the compressed electric cloud, and $N$ is the number of neutrino quanta that is determined from the decay scheme: $N=2$ for muon, 3 -for pion, and 21-for kaon. The masses of particles and characteristic sizes $R_{m}$ and $\rho_{m}$ are determined in general case at minimization of virtual rest-energy (5) on $R$ and $\rho$. Calculated in [3] values of the masses of $e, \mu, \pi^{0}$ and $K^{0}$ are in relation 0.547: 105.707: 134.963: $493.87(\mathrm{MeV})$ (by attachment to mass of neutral pion), that is in accord with experience data. It is shown that the masses of all considered particles, as well as for an electron, are proportional to the square of their equilibrium size $R_{m}$ :

$$
M c^{2}=f c^{2} R_{m}^{2}=12 \sigma \pi R_{m}^{2}
$$

It is assumed in the Standard Model that the masses of row of elementary particles can be represented in the form:

$$
M c^{2}=\lambda_{M} H / \sqrt{2}
$$

where $H=246 \mathrm{GeV}$ is the characteristic energy in the model of Higgs [4] [5], $\lambda_{M}$ is a dimensionless factor, characteristic for a certain particle with mass of $M$. We can make compatible formulas (6) and (7) for the masses, if preliminary we equate right parts of Equations (6) and (7); that results to:

$$
\lambda_{M}=R_{m}^{2} /[H /(\sqrt{2} \cdot 12 \sigma \pi)]=R_{m}^{2} / R_{x}^{2}
$$

where unknown size $R_{x}=[H /(\sqrt{2} \cdot 12 \sigma \pi)]^{1 / 2}=1.11 \times 10^{-10} \mathrm{~cm}$, that is comparable to $1 / 2$ the Compton wavelength of electron $2 \pi \hbar / m_{e} c=2.43 \times 10^{-10} \mathrm{~cm}$.

The result of the Formula (6) was used in [6] for the calculation of neutrino masses $v_{e}, v_{\mu}$ and $v_{\tau}$, for which the square of their electromagnetic radius was found in works [7]-[9]. It is shown in them that neutrinos of all types have a complex internal structure as a consequence of the virtual transitions 
$v_{\ell} \leftrightarrow \ell^{-}+W^{+}, \tilde{v}_{\ell} \leftrightarrow \ell^{+}+W^{-}$, where the lepton index $\ell$ means $e, \mu$ or $\tau$; $W$ are intermediate vector bosons, which are carriers of the weak interaction with mass $M_{\mathrm{w}}=80.4 \mathrm{GeV}$ [10]. Taking into account such virtual transitions in [7]-[9], it is found that the square of the electromagnetic radius of neutrino is equal to:

$$
\left\langle r^{2}\left(v_{\ell}\right)\right\rangle=\left(3 G_{F} / 8 \sqrt{2} \pi^{2} \hbar c\right)\left[(5 / 3) \operatorname{Ln} \alpha+(8 / 3) \operatorname{Ln}\left(M_{W} / m_{\ell}\right)+\eta\right]
$$

where $G_{F}=1.43 \times 10^{-62} \mathrm{~J} \cdot \mathrm{m}^{3}$ is the weak interaction constant, $\alpha=e^{2} / \hbar c$, and numerical constant $\eta$ is $1-2$. For a mean value $\eta=1.5$ taking into account $m_{\mu} c^{2}=105.66 \mathrm{MeV}$ and $m_{\tau}=1777 \mathrm{MeV}$ from (9) it follows that the characteristic values of the squared neutrino radii are equal to:

$$
\left\langle r^{2}\left(v_{e}\right)\right\rangle \cong 3 \times 10^{-33} \mathrm{~cm}^{2},\left\langle r^{2}\left(v_{\mu}\right)\right\rangle \cong 1.3 \times 10^{-33} \mathrm{~cm}^{2},\left\langle r^{2}\left(v_{\tau}\right)\right\rangle \cong 4.2 \times 10^{-34} \mathrm{~cm}^{2}
$$

To define the masses of neutrino in [6] simple suppositions are made:

1. Although neutrinos do not have an electric charge, they seems to have small electrostatic energy due to that spacial distributions of diverse charges produced by virtual pairs $(\ell, W)$ are slightly different. In this case the electrostatic energy of neutrino has a value $U\left(v_{\ell}\right)=\delta\left(v_{\ell}\right) e^{2} / r$, where $r$ is a virtual electromagnetic radius of neutrino, $\delta\left(v_{\ell}\right)$ is an unknown small parameter related to the distribution of charges in a structure of $v_{\ell}$.

2. Virtual rest-energy of neutrino consists of the confining potential $W_{s}=\sigma 4 \pi R^{2}$ and electrostatic energy:

$$
E=\sigma 4 \pi r^{2}+\delta\left(v_{\ell}\right) e^{2} / r
$$

3. The value of $\sigma$ is identical for all neutrinos.

Similarly, as for an electron, mass of neutrino would be found at being of a minimum of virtual energy (11):

$$
m\left(v_{\ell}\right)=3(\pi \sigma)^{1 / 3}\left[\delta\left(v_{\ell}\right) e^{2}\right]^{2 / 3} / c^{2}
$$

but as a value $\delta\left(v_{\ell}\right)$ is unknown, for determination of the neutrino masses we will take advantage of theoretical results [7]-[9] for the square of electromagnetic radius of neutrino $\left\langle r^{2}\left(v_{\ell}\right)\right\rangle$ and of Formula (6). Thus, putting the found values (10) for $\left\langle r^{2}\left(v_{\ell}\right)\right\rangle$ in Formula (6) instead of $R_{m}^{2}$, we find that the masses of neutrino are equal to:

$$
m\left(v_{e}\right) c^{2} \cong 4.3 \times 10^{-2} \mathrm{eV}, \quad m\left(v_{\mu}\right) c^{2} \cong 2 \times 10^{-2} \mathrm{eV}, \quad m\left(v_{\tau}\right) c^{2} \cong 6 \times 10^{-3} \mathrm{eV}
$$

Similar values for three neutrino mass eigenstates $\left(v_{1}, v_{2}, v_{3}\right)$ were received in [11] on the basis of Super-Kamiokande experimental results [12], inventively solving system of two equations with 3 unknown quantities, if supposing the case of inverted mass spectrum.

Values $\lambda\left(v_{\ell}\right)$ for a neutrino also can be found from Formula (8) at the substitution of values $\left\langle r^{2}\left(v_{\ell}\right)\right\rangle$ instead of $R_{m}^{2}$ :

$$
\lambda\left(v_{e}\right) \cong 2.47 \times 10^{-13}, \lambda\left(v_{\mu}\right) \cong 1.08 \times 10^{-13}, \lambda\left(v_{\tau}\right) \cong 3.4 \times 10^{-14}
$$

We will notice that as $G_{F} \cong \pi \alpha(\hbar c)^{3} /\left(\sqrt{2} \sin ^{2} \theta_{W} M_{W}^{2} c^{4}\right)$ [5] [13], taking into account Equations (4), (6) and (9) we get a general formula for the masses of neutrino in the form:

$$
m\left(v_{\ell}\right) \cong H(\ell) \alpha\left[m_{0} / M_{W}\right]^{2} m_{0}
$$

where a dimensionless factor $H(\ell)$ is equal to:

$$
H(\ell)=3^{-5} \pi^{-3} \sin ^{-2} \theta_{W}\left[(5 / 3) \operatorname{Ln} \alpha+(8 / 3) \operatorname{Ln}\left(M_{W} / m_{\ell}\right)+\eta\right]
$$

$\theta_{w}$-is the angle of Weinberg, $\left(\sin ^{2} \theta_{w}=0.23\right)$ and all 3 dimensionless coefficients: $H(\ell), \alpha$ and $\left[m_{0} / M_{W}\right]^{2}$ are much smaller than 1 . The values $\delta\left(v_{\ell}\right)$ are determined from Equation (12):

$$
\delta\left(v_{\ell}\right)=\left[m\left(v_{\ell}\right) c^{2}\right]^{3 / 2} /\left[3^{3 / 2} \alpha \hbar c(\pi \sigma)^{1 / 2}\right]
$$


and are equal to:

$$
\delta\left(v_{e}\right) \cong 1.10 \times 10^{-11}, \delta\left(v_{\mu}\right) \cong 3.17 \times 10^{-12}, \delta\left(v_{\tau}\right) \cong 5.6 \times 10^{-13}
$$

In Equation (5), also as in (1), the value $W_{S}=\sigma 4 \pi R^{2}$ plays a role of confining potential thanks to which the mass of the elementary particle is stabilized. This conception of the mass origin is simple and clear: complete internal energy is equal to $M c^{2}$; however, physical reason for the origin of confining potential is not clear. A model is offered in this article, explaining the origin of this potential and holding pressure due to the effect of impulse recoil of the special emitted virtual bosons.

So then for the ground of this model we will enter next suppositions:

1. By analogy with the model of Poincare we will suppose that every elementary particle has confining potential $\Phi(R)$, except for photons and gravitons. It is assumed that rest-energy of particle consists of confining potential, kinetic energy of internal motion, energy of the internal fields and electrostatic energy of the internal charges.

2. Every elementary particle radiates the special virtual bosons as spherical waves $A\left(\mathrm{e}^{i k r} / r\right) \mathrm{e}^{-i \omega t}$. Here $k$ is a wave-number of virtual boson, $\omega$ is its angular frequency, $r$ is distance from the center of a particle $(r \geq R), A$ is a normalizing constant not substantial for further consideration.

3. We will suppose that mass of such bosons $M_{H}$ is much more than masses of intermediate vector bosons $\mathrm{W}$ and $\mathrm{Z}^{0}$, where $\mathrm{Z}^{0}$ is a neutral carrier of the weak interaction with mass $91.2 \mathrm{GeV}$ [10]. Then a time of existence of such virtual bosons $\tau$ is much smaller than $2 \pi \hbar /\left(M_{W} c^{2}\right) \approx 5 \times 10^{-26}$ sec and distance of their run $L_{\tau}$ from the surface of elementary particle is much smaller than $2 \pi \hbar /\left(M_{W} c\right) \approx 2 \times 10^{-15} \mathrm{~cm}$.

4. Every elementary particle is the inexhaustible source of such virtual spherical waves, but the mass of the particle-source does not decrease, because virtual bosons through an instant $\tau$ return back into a source, due to interacting with the fields of vacuum.

5. We suppose that complete amount of the bosons emitted by a particle in a unit of time $N_{\mathrm{H}}$ is proportional to area of particle surface with a coefficient $\gamma$, characterizing intensity of radiation for the certain group of particles: $N_{H}=\gamma 4 \pi R^{2}$.

As every moving wave carries an impulse, it ensues from these suppositions, that on the surface of elementary particle because of the effect of impulse recoil, spherical waves are creating holding force of pressure $F(R)$ and confining potential

$$
\Phi(R)=\int F(R) \mathrm{d} R
$$

We will consider 2 cases for forms of boson spectrum:

a) the emitted bosons have a continuous spectrum of radiation in the interval of $k$ from 0 to $k_{\max }=K$ with the function of probability distribution $W_{a}(k, \xi)$, where $\xi=M_{H} c / \hbar$. As be shown, in this case confining potential is proportional to $R^{3}$.

b) the emitted bosons have a discrete spectrum of radiation : $k=\pi / R, 2 \pi / R, \cdots, n \pi / R, \cdots$ with the function of probability distribution $W_{b}(k, \xi)$. In this case the confining potential appears to be proportional to $R^{2}$.

Let's consider the case of a). We will enter the condition of normalizing for $W_{a}(k, \xi)$ so that

$\int_{0}^{K} W_{a}(k, \xi) \mathrm{d} k=1$. Then the number of impulses in an interval from $k$ to $k+\mathrm{d} k$ is equal to $N_{H} \cdot W_{a}(k, \xi) \mathrm{d} k=\gamma 4 \pi R^{2} W_{a}(k, \xi) \mathrm{d} k$, and complete force of pressure, operating on a surface $4 \pi R^{2}$ is equal to:

$$
F(R)=2 \int_{0}^{K} \hbar k \gamma 4 \pi R^{2} W_{a}(k, \xi) \mathrm{d} k
$$

Consequently confining potential for the case of a) is:

$$
\Phi_{3}(R)=R^{3}\left[(8 / 3) \pi \gamma \hbar \int_{0}^{K} k W_{a}(k, \xi) \mathrm{d} k\right]=R^{3}\left[(8 / 3) \pi \gamma \hbar k_{a v}\right]
$$


where $k_{a v}=\int_{0}^{K} k W_{a}(k, \xi) \mathrm{d} k$ is a quantum-mechanical mean value of $k$.

For the case of b) we will enter the condition of normalizing for $W_{b}(k, \xi): \sum_{n} W_{b}(n \pi / R, \xi)=1$. In this case total force of pressure on a surface $4 \pi R^{2}$ is equal to:

$$
F(R)=2 \sum_{n} \hbar(n \pi / R) \gamma 4 \pi R^{2} W_{b}(n \pi / R, \xi)
$$

The sum $\sum_{n}(n \pi / R) W_{b}(n \pi / R, \xi)$ in Formula (21) by definition is equal to $n_{a v} \cdot(\pi / R)$ where $n_{a v}$ is a quantum-mechanical mean value of $n$. As a result the confining potential $\Phi_{2}(R)$ is:

$$
\Phi_{2}(R)=R^{2}\left[4 \pi^{2} \gamma \hbar n_{a v}\right]
$$

Thus, in the article it's assumed that every elementary particle produces the special bosonic field that is present only in a thin layer at the surface of a particle. It is shown that this field can create the confining potential, stabilizing the mass of particle during the time of its life.

\section{References}

[1] Dirac, P.A.M. (1962) Proceedings of the Royal Society of London, Series A, Mathematical and Physical Sciences, 268, 57-67. http://dx.doi.org/10.1098/rspa.1962.0124

[2] Feynman, R., Leighton, R. and Sands, M. (1964) The Feynman Lectures on Physics, Vol. 2. Addison Wesley Pub. Com., Massachusetts, Palo Alto and London.

[3] Buravov, L.I. (2009) Russian Physics Journal, 52, 25-32. http://dx.doi.org/10.1007/s11182-009-9196-5

[4] Quigg, C. (2007) http://arxiv.org/pdf/0704.2232.pdf

[5] Vysotsky, M.I. (2011) Physics of Particles and Nuclei Letters, 8, 617-650. http://dx.doi.org/10.1134/S1547477111070181

[6] Buravov, L.I. (2013) Russian Physics Journal, 55, 1368-1369. http://dx.doi.org/10.1007/s11182-013-9968-9

[7] Bernstein, J. and Lee, T.D. (1963) Physical Review Letters, 11, 512-516. http://dx.doi.org/10.1103/PhysRevLett.11.512

[8] Lee, T.D. and Sirlin, A. (1964) Reviews of Modern Physics, 36, 666-669. http://dx.doi.org/10.1103/RevModPhys.36.666

[9] Cheng, W.K. and Bludman, S.A. (1964) Physical Review, 136B, 1787-1790. http://dx.doi.org/10.1103/PhysRev.136.B1787

[10] Particle Data Group (2012) Reviews, Tables, Plots.

[11] Hajdukovic, D.S. (2011) http://arxiv.org/abs/1106.5810

[12] Nakamura, K. and Petcov, S.T. (2010) Journal of Physics G, 37, 164-183.

[13] Prokhorov, A.M., Ed. (1992) Physics Encyclopedia, Vol. 5. Sovetskaya Entsiklopedia, Moscow. (In Russian) 